\title{
Bactericidal effects of non-thermal argon plasma in vitro, in biofilms and in the animal model of infected wounds
}

\author{
Svetlana A. Ermolaeva, ${ }^{1}$ Alexander F. Varfolomeev, ${ }^{1}$ \\ Marina Yu. Chernukha, ${ }^{1}$ Dmitry S. Yurov, ${ }^{1}$ Mikhail M. Vasiliev, ${ }^{2}$ \\ Anastasya A. Kaminskaya, ${ }^{1}$ Mikhail M. Moisenovich, ${ }^{3}$ Julia M. Romanova, ${ }^{1}$ \\ Arcady N. Murashev, ${ }^{4}$ Irina I. Selezneva, ${ }^{5}$ Tetsuji Shimizu, ${ }^{6}$ \\ Elena V. Sysolyatina, ${ }^{1}$ Igor A. Shaginyan, ${ }^{1}$ Oleg F. Petrov, ${ }^{2}$ \\ Evgeny I. Mayevsky, ${ }^{5}$ Vladimir E. Fortov, ${ }^{2}$ Gregor E. Morfill, ${ }^{6}$ \\ Boris S. Naroditsky ${ }^{1}$ and Alexander L. Gintsburg ${ }^{1}$ \\ ${ }^{1}$ Gamaleya Research Institute of Epidemiology and Microbiology, Russian Academy of Medical \\ Sciences, Moscow, Russia \\ ${ }^{2}$ Joint Institute of High Temperatures, Russian Academy of Sciences, Moscow, Russia \\ ${ }^{3}$ Lomonosov Moscow State University, Moscow, Russia \\ ${ }^{4}$ Shemyakin and Ovchinnikov Institute of Bioorganic Chemistry, Russian Academy of Sciences, \\ Pushchino, Russia \\ ${ }^{5}$ Institute of Theoretical and Experimental Biophysics, Russian Academy of Sciences, Pushchino, \\ Russia \\ ${ }^{6}$ Max Planck Institute for Extraterrestrial Physics, Munich, Germany
}

Correspondence Svetlana A. Ermolaeva sveta@ermolaeva.msk.su

Received 9 March 2010 Accepted 7 September 2010
Non-thermal (low-temperature) physical plasma is under intensive study as an alternative approach to control superficial wound and skin infections when the effectiveness of chemical agents is weak due to natural pathogen or biofilm resistance. The purpose of this study was to test the individual susceptibility of pathogenic bacteria to non-thermal argon plasma and to measure the effectiveness of plasma treatments against bacteria in biofilms and on wound surfaces. Overall, Gram-negative bacteria were more susceptible to plasma treatment than Gram-positive bacteria. For the Gramnegative bacteria Pseudomonas aeruginosa, Burkholderia cenocepacia and Escherichia coli, there were no survivors among the initial $10^{5}$ c.f.u. after a 5 min plasma treatment. The susceptibility of Gram-positive bacteria was species- and strain-specific. Streptococcus pyogenes was the most resistant with $17 \%$ survival of the initial $10^{5}$ c.f.u. after a 5 min plasma treatment. Staphylococcus aureus had a strain-dependent resistance with 0 and $10 \%$ survival from $10^{5}$ c.f.u. of the Sa 78 and ATCC 6538 strains, respectively. Staphylococcus epidermidis and Enterococcus faecium had medium resistance. Non-ionized argon gas was not bactericidal. Biofilms partly protected bacteria, with the efficiency of protection dependent on biofilm thickness. Bacteria in deeper biofilm layers survived better after the plasma treatment. A rat model of a superficial slash wound infected with $P$. aeruginosa and the plasma-sensitive Staphylococcus aureus strain Sa 78 was used to assess the efficiency of argon plasma treatment. A 10 min treatment significantly reduced bacterial loads on the wound surface. A 5-day course of daily plasma treatments eliminated $P$. aeruginosa from the plasma-treated animals 2 days earlier than from the control ones. A statistically significant increase in the rate of wound closure was observed in plasma-treated animals after the third day of the course. Wound healing in plasma-treated animals slowed down after the course had been completed. Overall, the results show considerable potential for non-thermal argon plasma in eliminating pathogenic bacteria from biofilms and wound surfaces. 


\section{INTRODUCTION}

Opportunistic bacterial pathogens are ubiquitous inhabitants of both the environment and the human body, causing serious infections in immunocompromised patients. Bacteria such as Staphylococcus aureus, Streptococcus species and Pseudomonas aeruginosa are common causative agents of skin and wound infections (Brook \& Frazier, 1998). Antibiotic therapy often fails to overcome opportunistic infections due to the widespread antibiotic resistance among pathogens (Rice, 2009; Strateva \& Yordanov, 2009). Biofilms formed by attached bacteria further complicate bacterial eradication (Davey \& O’Toole, 2000; Lynch \& Robertson, 2008).

To fight opportunistic infections, most studies have focused on finding chemical bactericidal agents. Application of physical treatments presents an alternative approach when the effectiveness of chemical agents is weak due to natural pathogen or biofilm resistance. One physical treatment with a microbicidal action is non-thermal (low-temperature) physical plasma (Kayes et al., 2007; Lee et al., 2006; Venezia et al., 2008). The non-thermal plasma is a flow of a partially ionized neutral gas of ambient macroscopic temperature, obtained at atmospheric pressure (Kong et al., 2009; Moreau et al., 2007, 2008). A plasma torch includes electrons, ions, free radicals and excited molecules, as well as UV radiation (Hury et al., 1998; Lassen et al., 2005; Lerouge et al., 2000a; Purevdorj et al., 2003; Shimizu et al., 2008). The plasma antimicrobial effect is due to the synergistic action of the reactive particles of the torch and UV, which causes oxidative stress and DNA damage (Kong et al., 2009; Moisan et al., 2001; Moreau et al., 2007; Sharma et al., 2009; Shimizu et al., 2008).

Non-thermal plasma can be effective for sterilization of medical equipment when heat inactivation is not suitable (Lee et al., 2006; Moisan et al., 2001; Venezia et al., 2008). The application of low-temperature plasma for skin regeneration in cosmetology was reported by Bogle (2006) and Elsaie \& Kammer (2008). A microbicidal effect was found when plasma was applied to contaminated dentin slices (Rupf et al., 2010). Its non-specific and penetrating mode of action allows consideration of the applicability of non-thermal plasma for local disinfection of tissues.

We have verified the applicability of non-thermal argon plasma in disinfection of wound surfaces. The bactericidal activity of argon plasma against important agents of wound-associated infections including $P$. aeruginosa, Staphylococcus aureus and Streptococcus pyogenes was tested in vitro, followed by testing of the effectiveness of plasma treatment against bacteria in biofilms and superficial nape wounds in rats.

\section{METHODS}

Bacterial strain and growth conditions. The bacterial strains tested are listed in Table 1. Eight of the strains were clinical isolates collected from Moscow hospitals in 2002-2009 (Avetisian et al., 2009; Shaginian et al., 2010), two were obtained from type collections and one was commercially available (Table 1). Burkholderia cenocepacia strain Bc 46 was derived from B. cenocepacia clinical isolate Bc 370 (Shaginian et al., 2003). Bc 46 hyperproduced biofilms due to a plasposon mutation in the gene encoding the global regulatory protein TetR (Romanova et al., 2009).

Bacteria were routinely cultivated on blood agar plates at $28{ }^{\circ} \mathrm{C}(P$. aeruginosa and B. cenocepacia) or $37^{\circ} \mathrm{C}$ (other species).

Biofilm growth. The Gram-negative bacteria B. cenocepacia strain Bc 46 and $P$. aeruginosa strain PA103 were used to grow biofilms. In general, the bacteria were grown in biofilms as described previously (Kaminskaya et al., 2007). Briefly, an overnight culture was diluted 1000 -fold in Luria-Bertani broth and cultured without agitation at $28{ }^{\circ} \mathrm{C}$ for $72 \mathrm{~h}$. A coverglass of $1.5 \mathrm{~cm}^{2}$ was placed vertically into the flask to allow a biofilm to form on its surface. The glass was removed after $72 \mathrm{~h}$, washed carefully with PBS and the biofilms were treated as described below.

Animal infection. Experiments on animals were conducted with the approval of the Animal Care and Use Committee of Gamaleya Institute of Epidemiology and Microbiology. Adult male Sprague Dawley rats of 560-700 g were anaesthetized with a mixture of $100 \mu \mathrm{l}$ Xyla (xylazine, $20 \mathrm{mg} \mathrm{ml}^{-1}$; Interchemie) and $100 \mu \mathrm{l}$ Zoletil 50 (Virbac). Wounds were made on rat napes by removing a piece of skin and subcutaneous oedemas with a sterile scalpel. Fresh wounds were covered with cotton pellets impregnated with a $1 \mathrm{ml}$ suspension of a mixed culture of $P$. aeruginosa PA103 $\left(10^{8}\right.$ c.f.u. $\left.\mathrm{ml}^{-1}\right)$ and Staphylococcus aureus Sa $78\left(10^{8}\right.$ c.f.u. $\left.\mathrm{ml}^{-1}\right)$, fixed in place with adhesive plaster for 3 days before being removed.

Sample treatment by non-thermal argon plasma or nonionized argon gas. All experiments were performed with a MicroPlaSter $\beta$ device (Shimizu et al., 2008) that allowed the researchers to use two regimes - an argon plasma and a placebo regime - with a flow of non-ionized argon gas. The plasma source is shown in Fig. 1. The argon plasma torch was $\sim 5 \mathrm{~cm}$ in length and $3.5 \mathrm{~cm}$ in diameter. During all experiments, the wound surfaces were treated at a distance of $2 \pm 0.2 \mathrm{~cm}$ with the plasma source at a gas temperature of $36 \pm 2{ }^{\circ} \mathrm{C}$.

Pure bacterial culture treatment. Tenfold dilutions of the bacterial suspension in PBS were plated on blood agar plates at $10^{3}-10^{5}$ c.f.u. per plate. Bacteria were treated with plasma or non-ionized argon gas for 2, 5 or $10 \mathrm{~min}$. Controls were left untreated. The plates were incubated for $72 \mathrm{~h}$ (Streptococcus pyogenes) or $48 \mathrm{~h}$ (other bacteria), and bacterial colonies were counted with a colony counting device (Schuett-Biotec). The effectiveness of the treatment was calculated as the percentage of c.f.u. observed on treated plates relative to c.f.u. on untreated plates at the lowest dilution where survival was observed.

Biofilm treatment and assessment. Biofilms cultured on coverglasses as described above were used in these experiments. For a qualitative assessment, glasses with cultured $P$. aeruginosa biofilms were placed on agar and treated with plasma or argon gas for $5 \mathrm{~min}$, following which they were transferred to sterile PBS and incubated at a room temperature for $2 \mathrm{~h}$ to complete bacterial killing. Thereafter, the biofilms were labelled using a Live/Dead Cell Viability Assay kit (Invitrogen) according to the manufacturer's instructions. Digital images were acquired with a confocal laser-scanning microscope (Zeiss Axiovert 200M LSM 510 META) as described by Kaminskaya et al. (2007).

For quantitative assays, biofilms formed by B. cenocepacia strain Bc 46 were scraped with a sterile blade, transferred to PBS and partly disrupted by pipetting. The suspension was divided into two portions. 
Table 1. Bacterial strains used in the study

\begin{tabular}{|c|c|c|c|c|c|}
\hline Species & Strain & Genotype & Resistance to:* & Source & Reference/supplier \\
\hline \multirow[t]{2}{*}{ P. aeruginosa } & PA103 & Wild-type & $\mathrm{AZ}, \mathrm{I}$ & Type collection & Avetisian et al. (2009) \\
\hline & $\mathrm{Pa} 12$ & Wild-type & $\mathrm{AZ}, \mathrm{G}, \mathrm{L}, \mathrm{T}, \mathrm{CX}, \mathrm{CZ}$ & Clinical isolate & Avetisian et al. (2009) \\
\hline \multirow{2}{*}{$\begin{array}{l}\text { Staphylococcus } \\
\text { aureus }\end{array}$} & ATCC Sa 6538 P & Wild-type & $\mathrm{NF}$ & Type collection & ATCC \\
\hline & Sa 78 & Wild-type & $\mathrm{A}$ & Clinical isolate & Shaginian et al. (2010) \\
\hline B. cenocepacia & Bc 46 & $\begin{array}{l}\text { Insertion in the tet } R \\
\text { gene, biofilm } \\
\text { hyperproducer }\end{array}$ & $\mathrm{ND}$ & $\begin{array}{l}\text { Derivative of the } \\
\text { clinical isolate } \\
\text { Bc } 370\end{array}$ & $\begin{array}{l}\text { Romanova et al. } \\
\text { (2009); Shaginian } \\
\text { et al. }(2003)\end{array}$ \\
\hline Escherichia coli & JM 109 & $\begin{array}{l}\mathrm{F}^{\prime} \text { traD36 proAB } \\
\text { lacl }^{\mathrm{q}} \Delta(\text { lacZ }) \mathrm{M} 15 / \text { endA1 } \\
\text { recA1 gyrA96 }\left(\mathrm{Nal}^{\mathrm{r}}\right) \text { thi } \\
\text { hsdR17 }\left(\mathrm{rk}^{-} \mathrm{mk}^{+}\right) \text {relA1 } \\
\text { supE44 } \Delta(\text { lac-proAB })\end{array}$ & $\mathrm{ND}$ & $\begin{array}{l}\text { Commercially } \\
\text { available }\end{array}$ & Fermentas \\
\hline Streptococcus pyogenes & Str 861 & Wild-type & $\mathrm{ND}$ & Clinical isolate & Shaginian et al. (2010) \\
\hline Enterococcus faecium & Ef 42 & Wild-type & $\mathrm{ND}$ & Clinical isolate & Bukharin et al. (1997) \\
\hline
\end{tabular}

${ }^{\star}$ A, Ampicillin; AZ, azithromycin; CX, cefotaxime; CZ, ceftazidime; E, erythromycin; G, gentamicin; I, imipenem; L, levomycetin; T, tobramycin. NF, Not found; ND, not done.

One was applied to the surface of a sterile Petri dish to form a layer of $\sim 1 \mathrm{~mm}$, which was given plasma or non-ionized argon gas treatment for $5 \mathrm{~min}$ as described above. As a control, the other sample was left untreated. Both samples were totally disaggregated by intensive mixing with an ultrasound disintegrator at $5 \mathrm{~W}$ for $7 \mathrm{~s}$ and serial dilutions were plated on blood agar plates. The biofilm disruption was controlled by bacterial visualization after application of the Gram staining method (data not shown).

Wound treatment and bacterial load assessment. During all procedures, animals were anaesthetized as described above. A convenient superficial swab sampling technique was used to collect specimens from the surface of infected wounds after removing the scabs. Swabs were placed in sterile PBS and serial dilutions of the bacterial suspension were plated on selective media to count specific pathogens. Cetrimide agar and tellurite-glycine agar (HiMedia) were used to count the number of P. aeruginosa and Staphylococcus aureus, respectively. Subsequently, animals were treated with the plasma torch for 5 or $10 \mathrm{~min}$ as described above, and the sampling was repeated. As controls, animals were treated with non-ionized argon for 5 or $10 \mathrm{~min}$ before sampling.

A 10 min daily course of plasma treatments was applied to a group of seven animals for 5 days to measure the effect of the procedure on
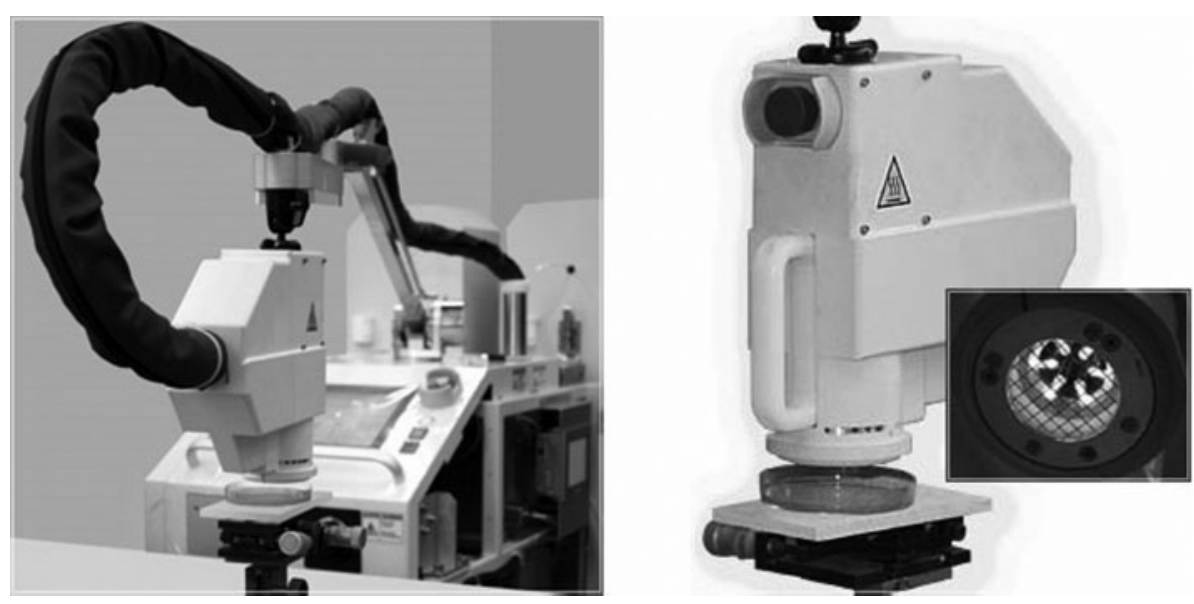

Fig. 1. Position of the plasma source relative to the sample, with the plasma torch shown on the right. 
wound healing. A control group of seven animals was treated with non-ionized argon gas. After treatment, the wounds were covered with a sterile medical membrane (Biokol). Samples were taken on days $1,3,5,8$ and 15 . Healing rates were monitored by measurement of the wound areas. Pictures of the wounds were taken every $24 \mathrm{~h}$ starting from the first day of treatment up to the last day of the procedure. Control pictures were taken on days 8,15 and 18. Wound areas were determined using UTHSCSA ImageTool v.3.0 software for picture processing.

Statistics. All experiments were performed using duplicate samples. To evaluate the data obtained in vitro, the mean values and SEM were calculated with Excel software (Miscrosoft Office 2003). The paired $t$ test included in the same software was used for assessment of statistical significance.

Stata 10 software (StataCorp LP) was used for numerical evaluation of bactericidal plasma effects in vivo. When bacterial counts on the wound surfaces were analysed, a noticeable dispersion of bacterial loads among animals was observed. Logarithmic data transformation was used to adjust numerical data with approximately normal distribution. The mean $\log$ difference, SEM and $95 \%$ confidence intervals (CIs) were calculated for the transformed data. Backtransforming the difference gave the geometric mean ratio and the CIs (Bland \& Altman, 1996).

\section{RESULTS AND DISCUSSION}

\section{Bactericidal effect of low-temperature plasma in vitro}

The initial study compared the individual susceptibility of micro-organisms belonging to different species and to different strains within the same species, using the most frequent causative agents of nosocomial and wound infections: P. aeruginosa, Staphylococcus aureus, Staphylococcus epidermidis, Streptococcus pyogenes, Escherichia coli and Enterococcus faecium (Table 1). The majority of strains were clinical isolates. The antibiotic resistance of the P. aeruginosa and Staphylococcus aureus strains has been characterized previously (Table 1).

Susceptibility to the argon plasma treatment was determined by applying plasma to planktonic bacterial cells plated on nutritive agar plates. To check the effect of nonionized argon gas, it was applied to parallel samples (see Methods). As a control, bacterial plates were left untreated. The bactericidal effect was determined as the percentage of c.f.u. after treatment with plasma or non-ionized argon compared with the untreated control (Fig. 2).

All tested strains of the Gram-negative bacteria P. aeruginosa, Escherichia coli and B. cenocepacia had a similar susceptibility to the argon plasma treatment: $<1 \%$ of the input c.f.u. formed colonies after a 2 min treatment $(P<0.005)$ (Fig. 2). There were no survivors from the initial $10^{5}$ c.f.u. after a 5 min treatment. The susceptibility of Gram-positive bacteria was species- and strain-dependent. Staphylococcus aureus clinical isolate Sa 78 was almost as sensitive as the Gram-negative bacteria; there were no survivors of the initial $10^{5}$ bacteria after a 5 min treatment. Streptococcus pyogenes clinical isolate Str 861 and Staphylococcus aureus type collection strain ATCC 6538 (Sa 6538; Fig. 2) had the lowest responsiveness. Approximately 17 and $10 \%$ of Str 861 and Sa 6538 , respectively, survived a $5 \mathrm{~min}$ treatment, with only $5 \%$ of Streptococcus pyogenes forming colonies after a $10 \mathrm{~min}$ treatment $(P<0.05)$. No bactericidal effect was observed with the non-ionized argon gas.

The results obtained suggested that, in general, nonthermal argon plasma is more effective against Gramnegative than Gram-positive bacteria. The differences in cell-wall structure can be critical for the differential sensitivity of Gram-negative and Gram-positive bacteria.

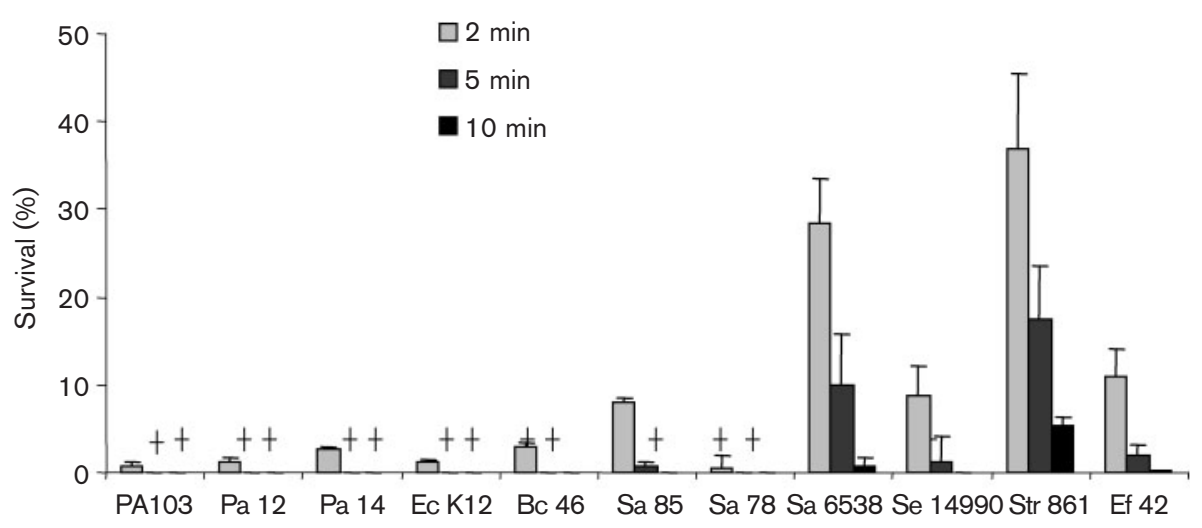

Strains

Fig. 2. Survival of pathogenic bacteria after plasma treatment. Bacteria were plated on nutritive agar and subjected to a 2,5 or $10 \mathrm{~min}$ exposure as indicated. The percentage of colonies formed after plasma treatment relative to untreated control plates is shown as percentage survival. Crosses indicate no survival of the input $10^{5}$ c.f.u. Pa, P. aeruginosa; Ec, Escherichia coli; Bc, $B$. cenocepacia; Sa, Staphylococcus aureus; Se, Staphylococcus epidermidis; Str, Streptococcus pyogenes; Ef, Enterococcus faecium (see Table 1 for strain details). Data represent means \pm SEM from three independent experiments undertaken in duplicate. 
Argon plasma acts via a complicated mechanism that includes a synergetic action of reactive species of different kinds, including ionized argon gas molecules, $\mathrm{O}_{\mathrm{x}}$ and $\mathrm{NO}_{\mathrm{x}}$ and UV light (Shimizu et al., 2008). A number of studies have demonstrated that bactericidal doses of UV light are comparable for Gram-negative and non-spore-forming Gram-positive bacteria (Chang et al., 1985; Sharp, 1939). Plasma reactive particles produce a general mechanical effect on the surface of living organisms, which has been called 'etching' (Chau et al., 1996; Lerouge et al., 2000a; Millard et al., 1976; Moisan et al., 2001; Moreau et al., 2008). Etching is due to the reaction of highly reactive gas radicals with organic materials, generating by-products that are subsequently desorbed from the surface. This causes perforations in the membranes of micro-organisms, which, in turn, remove the obstacle to secondary reactive species that might be formed in the medium. The effectiveness of etching depends on plasma composition (Lerouge et al., 2000b). The etching effect of the argon plasma may be less pronounced in Gram-positive bacteria, which have thick cell walls.

In contrast, the existence of highly sensitive and resistant strains belonging to the Gram-positive species Staphylococcus aureus, seen in the course of this study, suggests that a more complicated mechanism may be responsible for the natural resistance of certain bacteria to plasma treatment. This mechanism may include the effectiveness of the general SOS response and differences in membrane lipid and/or protein composition, making it important for further studies to reveal the details of plasma-caused damage and bacterial responses to it.

Bacterial spores are more resistant than vegetative bacterial cells to plasma (Hong et al., 2009; Kayes et al., 2007; Lee et al., 2006; Venezia et al., 2008). When non-spore-forming Gram-positive and Gram-negative bacteria were compared, the results were controversial. In some cases, no significant differences were detected (Kayes et al., 2007; Venezia et al., 2008), whilst other researchers found that Gram-positive bacteria were more resistant than Gram-negative bacteria (Lee et al., 2006). These discrepancies can be attributed to variation in the plasma devices used, and therefore in plasma compositions, producing different effects. However, the results obtained suggest that a strain-specific characteristic of the bacterial resistance may also be responsible for discrepancies in the results published previously, where most studies included a unique strain for the bacterial species (Kayes et al., 2007; Lee et al., 2006; Venezia et al., 2008). Further studies using a wider range of strains are required to determine whether the variability in resistance to plasma is characteristic only of Gram-positive pathogens and how strain-specific sensitivity depends on the plasma composition.

\section{Bactericidal effect of non-thermal plasma on bacteria in biofilms}

Compared with isolated bacterial cells, biofilms represent a major form of bacterial persistence on the surface of both medical equipment and chronic wounds (Lynch \& Robertson, 2008). In general, bacteria in biofilms are less sensitive to antimicrobial treatments (Davey \& O'Toole, 2000). The influence of biofilm formation on the receptiveness to plasma treatment was studied by taking the examples of the Gram-negative bacteria $B$. cenocepacia and $P$. aeruginosa.

To quantitatively assess the bactericidal effect of nonthermal plasma treatment, biofilms formed by B. cenocepacia strain Bc 46 were used. The Bc 46 strain overproduces biofilm biomass due to a mutation in the tet $R$ gene (Romanova et al., 2009). A plasma reduction of c.f.u. in biofilms was found in three independent experiments (data not given). However, the differences in survival rates shown in these experiments were much greater compared with those for isolated bacterial cells. The lowest level of survival was $0.005 \%$ from the initial bacterial load, whilst $2 \%$ of bacteria survived under the same conditions in another experiment $(P<0.05)$. Non-ionized argon gas caused no statistically significant changes in bacterial viability in biofilms (data not shown) or when it was applied to plated bacterial cultures.

Scattering in the data could be due to differences in bacterial survival at various biofilm layers, leading to the dependence of the bactericidal plasma effect on the thickness of a biofilm. To examine this suggestion and to gain further evidence of a bactericidal effect of argon plasma, we conducted a microscopic study of bacterial viability in various layers of biofilms formed by $P$. aeruginosa PA103. Experiments were performed with this strain, as biofilms formed by the B. cenocepacia strain Bc 46 varied significantly in thickness. Moreover, it was important to confirm the lethal effect of plasma on bacteria in biofilms for another bacterial model.

Plasma- or non-ionized-argon-treated $P$. aeruginosa biofilms were labelled with a Live/Dead Cell Viability Assay kit providing a two-colour fluorescence assay of bacterial cells based on membrane integrity (Fig. 3). Dead (red-labelled) bacteria prevailed in the plasma-treated biofilms, whilst the argon-treated biofilms included more live (green-labelled) than dead bacteria. Moreover, plasma-treated biofilms had higher concentrations of live bacteria at deeper layers compared with control, non-ionized-gas-treated biofilms. The presence of dead bacteria in argon-treated biofilms was not related to an effect of non-ionized argon gas. Dead bacteria were also seen in control non-treated biofilms (data not shown) and their appearance seemed to be due to the experimental conditions used for biofilm growth. The occurrence of dead bacteria within model biofilms grown under stationary conditions has been shown previously (Takenaka et al., 2001).

The results obtained indicated that the bacteria in biofilms were susceptible to the argon plasma, but that the effectiveness of the treatment was different from the top to the bottom of the bacterial layers and may therefore decrease in proportion to biofilm thickness. Our data are 

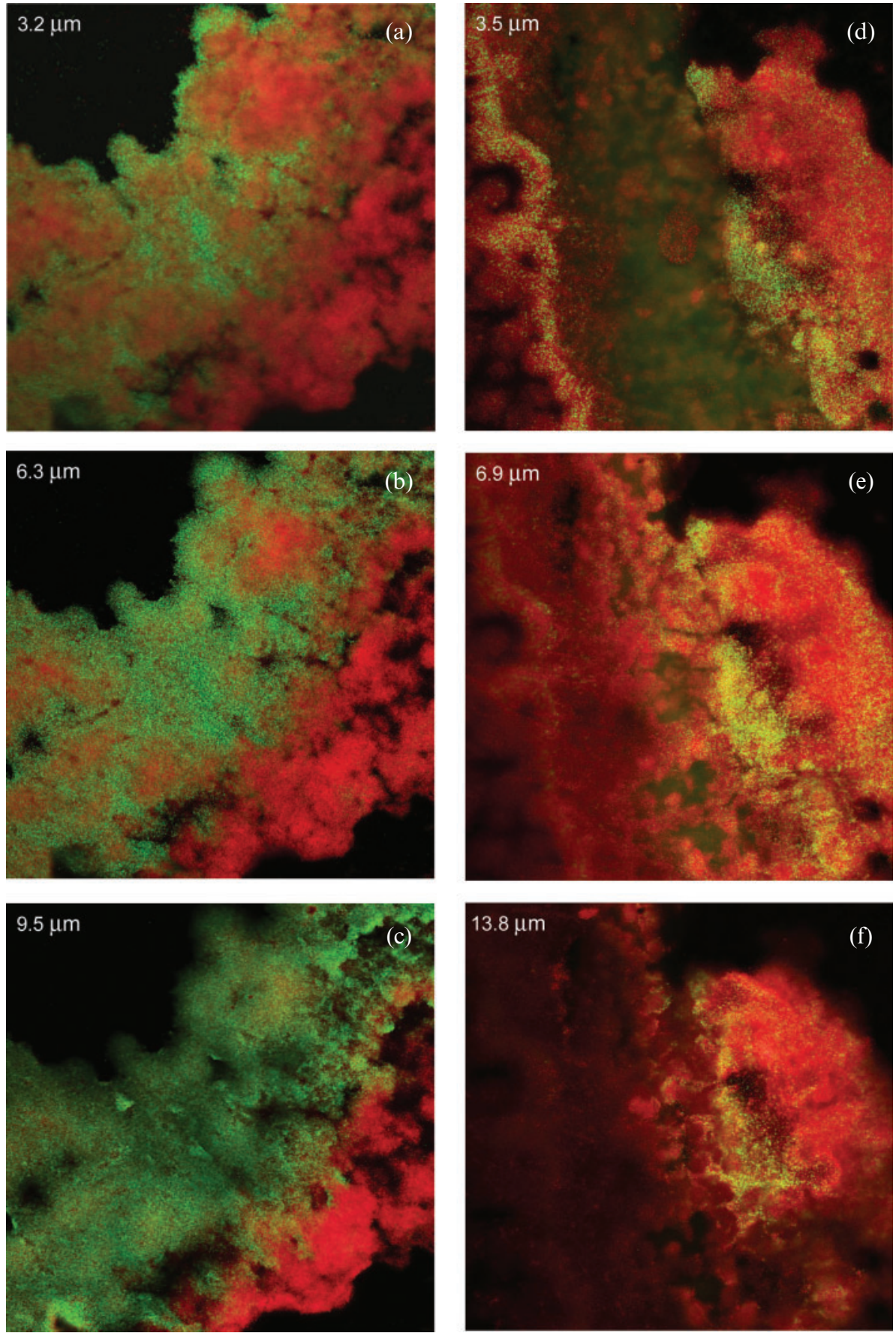

Fig. 3. Survival of $P$. aeruginosa PA103 in biofilms after plasma treatment. Biofilms formed on glass surfaces were treated with argon plasma or non-ionized argon for $5 \mathrm{~min}$. The cells were labelled with the fluorescent dyes of a Live/Dead Cell Viability Assay kit and visualized with a confocal laser-scanning microscope. Three consecutive sections for the control (a-c) and plasma-treated $(d-f)$ biofilms are shown. Live bacteria were stained green and dead bacteria red. The distance from the glass surface is shown in the upper left corner of each section.

consistent with previously published results that demonstrated inactivation of up to $99.6 \%$ of Chromobacterium violaceum used as a model Gram-negative micro-organism after a 5 min treatment with gas-discharge plasma applied to the biofilm (Joaquin et al., 2009). The susceptibility to plasma treatment of Gram-positive bacteria in biofilms was not addressed in this study. Nevertheless, one can suggest that some weakening of plasma bactericidal effects would be observed for biofilms versus planktonic bacterial cells for both Gram-negative and Gram-positive bacteria. 
Indeed, a study of the susceptibility of the Gram-positive bacterium Staphylococcus epidermidis to gliding discharge plasma demonstrated that diverse time frames are necessary to reach a similar bactericidal effect against planktonic bacteria and bacteria in biofilms: a reduction of 6 log units in $15 \mathrm{~min}$ for planktonic cells and in $70 \mathrm{~min}$ for biofilm cells (Kamgang et al., 2007).

\section{Effect of non-thermal plasma on Staphylococcus aureus and $\boldsymbol{P}$. aeruginosa infection in rats}

To evaluate the antimicrobial effectiveness of plasma treatments in vivo, a rat model of superficial slash wound infection was developed. Fresh nape wounds obtained in adult Sprague Dawley rats were seeded with a mixed bacterial suspension of the plasma-sensitive Staphylococcus aureus strain Sa 78 and the P. aeruginosa strain PA103 $\left(10^{8}\right.$ c.f.u. of each), as described in Methods. An acute wound infection developed in 3 days producing local inflammation, oedema and pus (data not shown).

Counting of total pathogens before treatment, as determined by a superficial sampling, ranged from $1 \times 10^{3}$ to $1 \times 10^{5}$ c.f.u. per sample for $P$. aeruginosa and from $2 \times 10^{3}$ to $6 \times 10^{5}$ c.f.u. per sample for Staphylococcus aureus. The bactericidal effect of plasma was expressed as a percentage of surviving c.f.u. for each pathogen (Fig. 4). After a $5 \mathrm{~min}$ treatment, the mean survival was $\sim 100 \%(P<0.05)$, which compared with the results obtained for the control animals treated with non-ionized argon gas with a placebo regimen.

After a 10 min plasma treatment, the percentage of surviving c.f.u. observed in various animals ranged from 0.1 to $\sim 100 \%$ for P. aeruginosa PA103, and from 1.2 to $\sim 100 \%$ for Staphylococcus aureus Sa 78. For P. aeruginosa, the value for the geometric mean ratio was $0.58 \%$ (95\% CI $0.3-100 \% ; P=0.055)$. For Staphylococcus aureus, the value for the geometric mean ratio was $6.4 \%(95 \%$ CI $0.8-50.0 \%$;
$P<0.05)$. A $10 \mathrm{~min}$ treatment with non-ionized argon gas caused no substantial deviations in bacterial load.

Thus, a comparable bactericidal effect for bacterial cells in vitro, biofilms and wound communities after 2, 5 and 10 min treatments was obtained (compare Figs 2 and 4). Isbary et al. (2010) used nitrocellulose filters to evaluate bacterial loads when reporting on the bactericidal effect of a 5 min treatment with argon plasma of patients with chronically infected wounds. The swab technique might partly mask the bactericidal effect on the wound surface, and we found no effect on bacterial load after a $5 \mathrm{~min}$ treatment. The deep bacterial location inside wound tissues, biofilm formation and specific composition of wound liquids might also lead to a weakening of the bactericidal effect of plasma in vivo. Nevertheless, our results indicated that bacteria on wound surfaces remained susceptible to plasma treatment, although more time was needed to inactivate them.

To assess the potential of argon plasma as a wound-healing agent, a 5-day course was applied starting from day 3 after infection. The course included a $10 \mathrm{~min}$ treatment each day. The control animals were treated with non-ionized argon gas. $P$. aeruginosa was totally eliminated from the wound surfaces after 3 days of application of the argon plasma. In samples taken from control animals, $P$. aeruginosa was recovered for a further 2 days compared with plasma-treated animals. However, we failed to eliminate Staphylococcus aureus during a 5-day plasma course, and Staphylococcus aureus bacteria were isolated on day 8 after initiation of the course.

In the wound areas measured every day during the course and on days 8,15 and 18 after the course had been started, a statistically significant increase in closure rate was seen in the plasma-treated animals starting from day 3 up to day 5 (last day) of the course $(P<0.05$; Fig. 5$)$. Wound closure was observed starting from day 13 for both control and plasma-treated animals. Total closure of the wound took
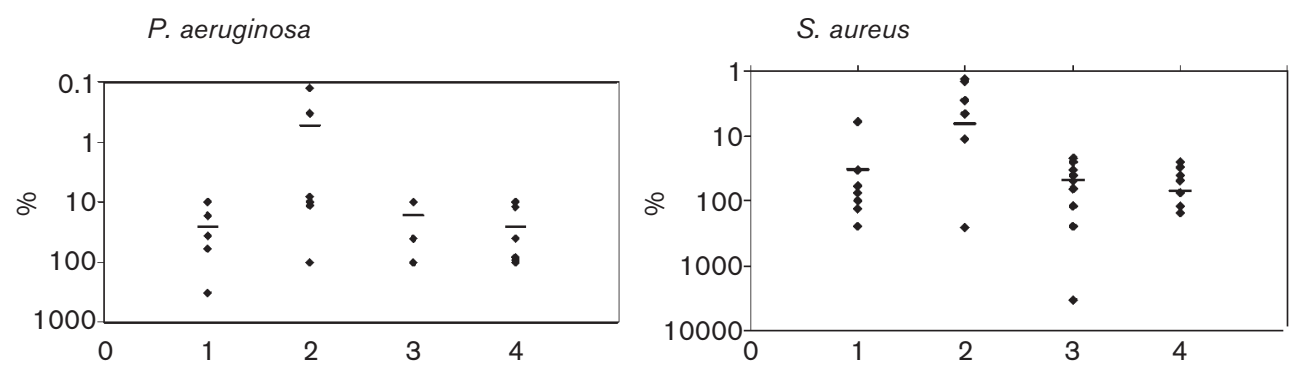

Fig. 4. Bactericidal effect of argon plasma on wound-associated bacteria. Wounds made on adult Sprague Dawley rat napes were seeded with $P$. aeruginosa PA103 and Staphylococcus aureus Sa 78 (10 8 c.f.u. each). Bacterial samples were collected with cotton swabs on day 3 after infection. Sampling was carried out before and after the plasma treatment applied for 5 or 10 min (1 and 2, respectively), or before and after a 5 or 10 min treatment with the non-ionized argon gas (3 and 4 , respectively). Bacteria were washed from the swabs into PBS and bacterial loads were determined after plating serial dilutions on pathogen-specific selective media. Data represent the percentage of bacterial counts after treatment in relation to the initial load of each animal. Mean values, represented by horizontal bars, were calculated for groups of seven animals. 


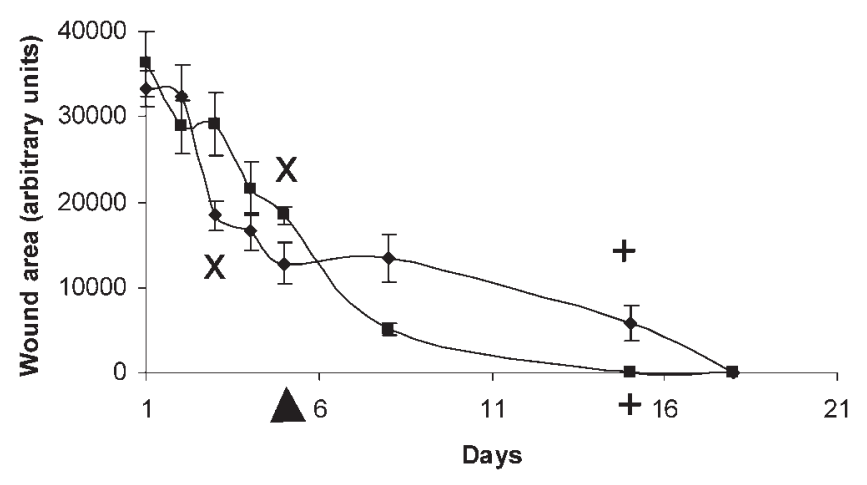

Fig. 5. Effect of a 5-day course of daily plasma treatment on wound closure in rats. The nape wound infection was as described in the legend to Fig. 4. The means \pm SEM of the wound areas measured, as described in Methods, are shown for groups of seven animals treated with plasma $(\boldsymbol{})$ or non-ionized $(\boldsymbol{\square})$ argon. Measurements were started on the day of the first procedure. The last day of plasma treatment is designated with an arrowhead. The day when each specific pathogen was eliminated from all animals is designated with an $\mathrm{X}$ and $\mathrm{a}+$ for $P$. aeruginosa and Staphylococcus aureus, respectively.

place on day 15 in the control animals. However, the process of wound healing slowed down in two of seven plasma-treated animals, whose wounds closed by day 18 .

The results obtained demonstrated that the plasma treatment was effective in eliminating $P$. aeruginosa and favoured improved wound healing (Fig. 5). In contrast, the delay in the wound closure observed in two animals after plasma treatment might have been due to incomplete elimination of Staphylococcus aureus or additional infection that was not recognized, as only specific pathogens were traced with selective media. Residual plasma effects on wound tissues also cannot be totally excluded. Although the plasma did not visibly damage the wound surface, direct plasma effects on animal tissues, and particularly on immune cells, which may change the wound healing dynamics, were not studied here. Non-thermal plasma does reduce the viability and induces apoptosis of tumour cells in vitro (Calugaru et al., 2005). To our knowledge, data concerning the results of plasma application on immune system activity and the cell regeneration process in wounds are not yet available.

Generally, the results demonstrated a considerable potential of non-thermal argon plasma in eliminating pathogenic bacteria both in vitro and in vivo. A comfortable temperature and a relatively large area of exposure with no special requirements for relief of the treated surfaces, in combination with bactericidal action against widespread microorganisms with multiple antibiotic resistance, allow us to conclude that non-thermal plasma is a highly advantageous sterilization method. Although the applicability of this approach to treatment of infected wounds requires more extensive studies, non-thermal plasma is a promising method for antimicrobial treatment of wounds infected with opportunistic bacteria where other approaches fail.

\section{ACKNOWLEDGEMENTS}

We thank ADTEC Plasma Technology Co. Ltd, and in particular Mr Urayama, for their part in the development of the MicroPlaSter device. The authors are grateful to Dr A. Valyshev and Dr I. Valysheva for E. faecium strain Ef 42 . The authors are extremely grateful to Professor M. Bland and Dr K. Belyaev for help with statistical analysis, and to Professor K. Mathee and the anonymous referees for valuable comments on the manuscript. This work was supported by the Ministry of Education and Science (grants N 14.740.11.0118 and 02.740.11.0310) and the Russian Fund for Basic Research (grant N 0808-12226).

\section{REFERENCES}

Avetisian, L. P., Chernukha, M. Y., Gabrielian, N. Y., Kovtun, N. A., Gorskaia, E. M. \& Shaginian, I. A. (2009). Genotypic features of Pseudomonas aeruginosa strains circulating in surgical hospital. $\mathrm{Zh}$ Mikrobiol Epidemiol Immunobiol 5, 33-38 (in Russian).

Bland, J. M. \& Altman, D. G. (1996). Transformations, means, and confidence intervals. BMJ 312, 1079.

Bogle, M. A. (2006). Plasma skin regeneration technology. Skin Therapy Lett 11, 7-9.

Brook, I. \& Frazier, E. H. (1998). Aerobic and anaerobic microbiology of infection after trauma. Am J Emerg Med 16, 585-591.

Bukharin, O. V., Valyshev, A. V., Elagina, N. N., Ivanov, I. B. \& Cherkasov, S. V. (1997). The photometric determination of the antilysozyme activity of microorganisms. Zh Mikrobiol Epidemiol Immunobiol 4, 117-120 (in Russian).

Calugaru, A., Cremer, L., Herold, A., Lupu, A., Szegli, G., Lungu, C., Lungu, A. \& Georgescu, N. (2005). The effect of the plasma needle on tumoral cell lines apoptosis. Roum Arch Microbiol Immunol 64, 57-64.

Chang, J. C., Ossoff, S. F., Lobe, D. C., Dorfman, M. H., Dumais, C. M., Qualls, R. G. \& Johnson, J. D. (1985). UV inactivation of pathogenic and indicator microorganisms. Appl Environ Microbiol 49, 1361-1365.

Chau, T. T., Kao, K. C., Blank, G. \& Madrid, F. (1996). Microwave plasmas for low-temperature dry sterilization. Biomaterials 17, 12731277.

Davey, M. E. \& O'Toole, G. A. (2000). Microbial biofilms: from ecology to molecular genetics. Microbiol Mol Biol Rev 64, 847-867.

Elsaie, M. L. \& Kammer, J. N. (2008). Evaluation of plasma skin regeneration technology for cutaneous remodeling. $J$ Cosmet Dermatol 7, 309-311.

Hong, Y. F., Kang, J. G., Lee, H. Y., Uhm, H. S., Moon, E. \& Park, Y. H. (2009). Sterilization effect of atmospheric plasma on Escherichia coli and Bacillus subtilis endospores. Lett Appl Microbiol 48, 33-37.

Hury, S., Vidal, D. R., Desor, F., Pelletier, J. \& Lagarde, T. (1998). A parametric study of the destruction efficiency of Bacillus spores in low pressure oxygen-based plasmas. Lett Appl Microbiol 26, 417-421.

Isbary, G., Morfill, G., Schmidt, H. U., Georgi, M., Ramrath, K., Heinlin, J., Karrer, S., Landthaler, M., Shimizu, T. \& other authors (2010). A first prospective randomized controlled trial to decrease bacterial load using cold atmospheric argon plasma on chronic wounds in patients. Br J Dermatol 163, 78-82.

Joaquin, J. C., Kwan, C., Abramzon, N., Vandervoort, K. \& BrellesMariño, G. (2009). Is gas-discharge plasma a new solution to the old problem of biofilm inactivation? Microbiology 155, 724-732. 
Kamgang, J. O., Briandet, R., Herry, J. M., Brisset, J. L. \& Naitali, M. (2007). Destruction of planktonic, adherent and biofilm cells of Staphylococcus epidermidis using a gliding discharge in humid air. J Appl Microbiol 103, 621-628.

Kaminskaya, A., Pushkareva, V., Moisenovich, M., Stepanova, T., Volkova, N., Romanova, J., Litvin, V., Gintsburg, A. \& Ermolaeva, S. (2007). Stimulation of biofilm formation by insertion of Tetrahymena pyriformis wells within Burkholderia cenocepacia biofilms. Mol Gen Microbiol Virol 22, 186-194 (erratum Mol Gen Microbiol Virol 23, 51).

Kayes, M. M., Critzer, F. J., Kelly-Wintenberg, K., Roth, J. R., Montie, T. C. \& Golden, D. A. (2007). Inactivation of foodborne pathogens using a one atmosphere uniform glow discharge plasma. Foodborne Pathog Dis 4, 50-59.

Kong, M. G., Kroesen, G., Morfill, G., Nosenko, T., Shimizu, T., van Dijk, J. \& Zimmermann, J. L. (2009). Plasma medicine: an introductory review. New J Phys 11, 115012.

Lassen, K. S., Nordby, B. \& Grün, R. (2005). The dependence of the sporicidal effects on the power and pressure of RF-generated plasma processes. J Biomed Mater Res B Appl Biomater 74, 553-559.

Lee, K., Paek, K., Ju, W. \& Lee, Y. (2006). Sterilization of bacteria, yeast, and bacterial endospores by atmospheric-pressure cold plasma using helium and oxygen. J Microbiol 44, 269-275.

Lerouge, S., Guignot, C., Tabrizian, M., Ferrier, D., Yagoubi, N. \& Yahia, L. (2000a). Plasma-based sterilization: effect on surface and bulk properties and hydrolytic stability of reprocessed polyurethane electrophysiology catheters. J Biomed Mater Res 52, 774-782.

Lerouge, S., Wertheimer, M. R., Marchand, R., Tabrizian, M. \& Yahia, L. (2000b). Effect of gas composition on spore mortality and etching during low-pressure plasma sterilization. J Biomed Mater Res 51, 128-135.

Lynch, A. S. \& Robertson, G. T. (2008). Bacterial and fungal biofilm infections. Annu Rev Med 59, 415-428.

Millard, M. M., Scherrer, R. \& Thomas, R. S. (1976). Surface analysis and depth profile composition of bacterial cells by $\mathrm{x}$-ray photoelectron spectroscopy and oxygen plasma etching. Biochem Biophys Res Commun 72, 1209-1217.

Moisan, M., Barbeau, J., Moreau, S., Pelletier, J., Tabrizian, M. \& Yahia, L. H. (2001). Low-temperature sterilization using gas plasmas: a review of the experiments and an analysis of the inactivation mechanisms. Int J Pharm 226, 1-21.

Moreau, M., Feuilloley, M. G. J., Veron, W., Meylheuc, T., Chevalier, S., Brisset, J. \& Orange, N. (2007). Gliding arc discharge in the potato pathogen Erwinia carotovora subsp. atroseptica: mechanism of lethal action and effect on membrane-associated molecules. Appl Environ Microbiol 73, 5904-5910.
Moreau, M., Orange, N. \& Feuilloley, M. G. J. (2008). Nonthermal plasma technologies: new tools for bio-decontamination. Biotechnol Adv 26, 610-617.

Purevdorj, D., Igura, N., Ariyada, O. \& Hayakawa, I. (2003). Effect of feed gas composition of gas discharge plasmas on Bacillus pumilus spore mortality. Lett Appl Microbiol 37, 31-34.

Rice, L. B. (2009). The clinical consequences of antimicrobial resistance. Curr Opin Microbiol 12, 476-481.

Romanova, I. M., Stepanova, T. V., Nesterenko, L. N., Balunets, D. V., Andreev, A. L., Shevliagina, N. V., Borovaia, T. G. \& Gintsburg, A. L. (2009). Persistence of Burkholderia cenocepacia bacteria in vivo in dependence of their ability to form biofilms. Zh Mikrobiol Epidemiol Immunobiol 4, 29-33 (in Russian).

Rupf, S., Lehmann, A., Hannig, M., Schäfer, B., Schubert, A., Feldmann, U. \& Schindler, A. (2010). Killing of adherent oral microbes by a nonthermal atmospheric plasma jet. J Med Microbiol 59, 206-212.

Shaginian, I. A., Khmel', I. A., Romanova, I. M., Veselova, M. A., Chernukha, M. I., Chernin, L. S., Sidorenko, S. V., Lipasova, V. A., Kovtun, V. P. \& other authors (2003). Clinical strains of Burkholderia cepacia: characteristic and detection of the components in the quorum sensing regulatory system. Mol Gen Mikrobiol Virusol 4, 15-20 (in Russian).

Shaginian, I. A., Kapranov, N. I., Chernukha, M. I., Alekseeva, G. V., Semykin, S. I., Avetisian, L. R., Kashirskaia, N. I., Pivkina, N. V., Danilina, G. A. \& other authors (2010). Microbial population of lower respiratory tract in children from different age groups with cystic fibrosis. Zh Mikrobiol Epidemiol Immunobiol 1, 15-20 (in Russian).

Sharma, A., Collins, G. \& Pruden, A. (2009). Differential gene expression in Escherichia coli following exposure to nonthermal atmospheric pressure plasma. J Appl Microbiol 107, 1440-1449.

Sharp, D. G. (1939). The lethal action of short ultraviolet rays on several common pathogenic bacteria. J Bacteriol 37, 447-460.

Shimizu, T., Steffes, B., Pompl, R., Jamitzky, F., Bunk, W., Ramrath, K., Georgi, M., Stolz, W., Schmidt, H.-U. \& other authors (2008). Characterization of microwave plasma torch for decontamination. Plasma Process Polym 5, 577-582.

Strateva, T. \& Yordanov, D. (2009). Pseudomonas aeruginosa - a phenomenon of bacterial resistance. J Med Microbiol 58, 1133-1148.

Takenaka, S., Iwaku, M. \& Hoshino, E. (2001). Artificial Pseudomonas aeruginosa biofilms and confocal laser scanning microscopic analysis. J Infect Chemother 7, 87-93.

Venezia, R. A., Orrico, M., Houston, E., Yin, S. \& Naumova, Y. Y. (2008). Lethal activity of nonthermal plasma sterilization against microorganisms. Infect Control Hosp Epidemiol 29, 430-436. 